\title{
MJN KNOWLEDGE AND PRACTICES OF OSTEOPOROSIS PREVENTIVE MEASURES AMONG WOMEN IN A TERTIARY TEACHING HOSPITAL
}

\author{
Hasni Embong*, Yew J. M. \\ School of Health Sciences, Health Campus, Kelantan, Universiti Sains Malaysia, Malaysia \\ *Corresponding Author's Email: ehasni@usm.my
}

\begin{abstract}
Introduction: Osteoporotic fracture is a major public health problem in the pre and peri-menopausal period, associated with substantial morbidity, socio-economic burden and mortality. Poor knowledge and practice related to osteoporosis have been noted worldwide. In Malaysia, few studies have measured the knowledge and practice of osteoporosis among women. Objectives: This study aimed to determine the knowledge and practices of osteoporosis preventive measures among women in Hospital USM. Methods: A cross-sectional study using a validated, self-administered questionnaire about knowledge and practices of osteoporosis measures was employed with 185 hospitalized women in Hospital USM. A simple random sampling was used for the recruitment of participants. The data were analyzed using Chi-Square tests. Results: The Majority of the participants, $67 \%$, belong to $45-55$ age group with a mean age of 50.35 years. The findings indicate $40.5 \%$ had a good level of knowledge towards osteoporosis, while 53.5\% had a poor practice level. Conclusion: Knowledge of osteoporosis and practices is still at a fair level, affecting osteoporosis prevention practices. Time was spend watching television. Therefore, public health strategies should also be developed in Malaysia to increase women's knowledge of osteoporosis prevention and change lifestyle habits.
\end{abstract}

Keywords: Osteoporosis; Knowledge, Prevention Practices; Malaysian Women

\section{INTRODUCTION}

Osteoporosis is the most common public health illness among women. Osteoporosis is also a disease of decreased bone mineral density that predisposes individuals to injuries, including minor falls or wounds (Tümay, Lale \& Nursel, 2017). Osteoporosis causes more than 8.9 million fractures annually globally, resulting in an osteoporotic fracture every 3 seconds (Bilal et al., 2017). The number of older people with fractures due to osteoporosis increases significantly as the ageing population increases significantly. It is estimated that 10 million Americans have osteoporosis (National Osteoporosis Foundation, 2015), and the people who are four times more likely to be affected are women. It has been estimated that more than 1 million Malaysians are at risk of developing osteoporosis, of which $20 \%$ are men, according to the Arthritis Foundation of Malaysia. In comparison, $51.8 \%$ of urban Malaysian women have near-menopause osteoporosis (Khan et al., 2014). About $13-18 \%$ of women aged 50 and over have osteoporosis, while for those over the age of 80 , the proportion increases to $70 \%$ (Puttapitakpong et al., 2014).
Factors have profound effects on the fitness, satisfaction and quality of life of a person due to osteoporosis, leading to chronic pain, long-term disability and death (International Osteoporosis Foundation, 2017). Osteoporosis may lead to serious complications following minor bone damage or mortality, including fractures of the spine, wrist, shoulder, pelvis and upper arm. After osteoporosis fractures such as experiencing discomfort, loss of freedom, adaptation concerns, and more serious worries about the future, the quality of life could also be affected (Martin et al., 2002). According to data released in 2018 by the World Health Organization (WHO), affected males and females are $73.2 \%$ and $77.6 \%$, respectively (WHO, 2018). This data showed that the life expectancy of the female is higher than male. The proportion of the population suffering osteoporotic fractures increases as our life expectancy increases.

Osteoporosis has many risk factors, such as age, gender, ethnicity, body mass index (BMI), genetics, diet, lifestyle, and family history (Ahmad et al., 2014). According to Samia et al., 2017, a sedentary lifestyle, insufficient calcium intake, heavy smoking, excessive 
alcohol consumption, low nutrition, menopausal women, aged over 50 or have a family history of osteoporosis with increased osteoporotic fractures. Puttapitakpoong et al. (2014) revealed that the daily recommendation for calcium intake to avoid osteoporosis could not be fulfilled by a significant proportion of the population. Furthermore, a study note that an unhealthy lifestyle can induce osteoporosis, such as smoking and drinking alcohol. According to studies by Abd-Alhameed et al. (2010), only a small percentage of postmenopausal women have been shown to take preventive steps, such as taking proper diet and consuming essential food supplements that can make healthy bones.

Globally, insufficient osteoporosis-related awareness and activities have been noted (De Silva et al., 2014). Many women may not have a good understanding of osteoporosis. Before making wise prevention decisions, they need to know how to avoid osteoporosis and take preventive steps (Terrio \& Auld, 2002). Efforts to raise public understanding of the severity, preventive measures and care of osteoporosis to minimise the frequency of osteoporosis are essential (Saw et al., 2003). These results point out that consciousness in community needs to be raised urgently to make them accept osteoporosis as one of the crucial health implications of their lives (AbdAlhameed, Saba \& Darwish, 2010).

The central component of effective educational intervention by healthcare practitioners is awareness of the disease and the extent to which they practise preventive behaviour (Vujasinovic-Stupar et al., 2017). There is a shortage of evidence in industrialised and developing countries on women's awareness and practise of osteoporosis and preventive measures. In order to direct public health campaigns, measuring the level of awareness in the community will help. Accelerated bone loss among postmenopausal women can occur or at least slow down the promotion of preventive measures and healthy behaviours. Indeed, women would not be able to recognise their own risk of contracting the disease and turn to health-related behaviours without sufficient information (Vujasinovic-Stupar et al., 2017).

It is therefore very important to undertake this research study to test the level of information, attitude, and practise among women 45 years of age and above about osteoporosis to raise awareness of the community's risk of osteoporosis. This study has the potential to open another window for the promotion of osteoporosis preventive measures in Malaysia, resulting in the facilitation of awareness campaigns and communitybased education on preventive measures.

\section{METHODOLOGY}

This Universiti Sains Malaysia research was accepted by the institutional review board. To evaluate the level of awareness and practise regarding osteoporosis among women in teaching hospitals in north-eastern peninsular Malaysia, a cross-sectional research design was used. The prevalence was estimated using the WHO sample size calculator at 50 percent with a confidence level of 95 percent, an expected population proportion $(p)$ of 0.3 , and absolute precision (d) of 0.05 . Using Microsoft Excel programme, simple random sampling was carried out to recruit qualified study participants (185). Participant research requirements included women aged 45 and above who were admitted to and voluntarily agreed to participate in the TCA clinic Hospital Universiti Sains Malaysia during the data collection period between June and July 2018. Any qualifying woman diagnosed with osteoporosis or any bone related disorders was included in the exclusion criteria.

Data collection consisted of distributing a selfadministered questionnaire to women aged 45 years old and above who visited the hospital, 185 of whom agreed to participate, signed the informed consent form, and completed the questionnaire. The questionnaire used in this study was adapted with permission from the previous researcher (Khan et al., 2014). The questionnaire consisted of three sections: (A) Sociodemographic data (age, ethnicity, marital status, social status, educational qualification, and economic status); (B) knowledge related to osteoporosis, (C) attitude to osteoporosis, and (3) practice assessing patient perceptions for osteoporosis.

The questionnaires were submitted to a panel of three experts to evaluate content validity, testing for format and item construction. All items were approved, assuring good content validity. A pilot study was carried out with 30 participants to refine the questionnaire. Its reliability was assessed using Cronbach's alpha (0.715). The first four questions of knowledge section were general questions related to osteoporosis, while a list of 10 most significant risk factor of osteoporosis were given in question 5. Question 6 comprises three most common complications of osteoporosis. The attitude and practice section of questionnaire consists of 10 questions each. Each correct answer in the knowledge domain carried 
one mark while wrong or don't know carried 0 mark. This gave a total score range of $0-17$ for knowledge section.

In the case of practice section, 'Never' was scored 0 while 'Rarely', 'Sometimes', 'Frequently' and 'Always' were scored as '1', '2', '3', and '4', respectively. 'Always' shows maximum frequency for an event, and therefore it was assigned the highest score. This gave a score range of 0 to 40 to practice section (Khan et al., 2014). The scores in knowledge and practice domains were categorized as poor (less than and equal to 50\%), fair (51 to $69 \%$ ) and good (70\% and above) (Khan et al., 2014).

Statistical analyses were conducted using the Statistical Package for Social Science (SPSS) statistical software (v.24.0). Descriptive statistics were used to describe frequency and percentage of central tendencies. The Chi-square test or Fisher's Exact test was used to determine the relationship between the sociodemographic data and knowledge and practice. The significance level of all tests was set at $\mathrm{a}<0.05$. All null hypotheses were rejected if $p$-value $<0.05$.

\section{RESULTS}

\section{Participant's Socio-demographic Characteristics}

Table 1 summarizes the socio-demographic characteristics of the 185 study participants. Participants' mean age was $50.35(6.583)$ years. The majority were Malay ethnicity $(87.0 \%)$, married $(81.1 \%)$, housewife $(60.0 \%)$, secondary education $(69.2 \%)$ and had economic status less than RM 1000 (58.4\%).

Table 1: Participant's Demographic Characteristics $(n=185)$

\begin{tabular}{|l|c|c|}
\hline Variables & n & (\%) Mean (SD) \\
\hline Age (years) & \multicolumn{2}{|c|}{$50.35(6.583)$} \\
\hline $45-55$ & 124 & $(67.0)$ \\
\hline $56-65$ & 48 & $(25.9)$ \\
\hline 66 and above & 13 & $(7.0)$ \\
\hline Ethnicity \\
\hline Malay & 161 & $(87.0)$ \\
\hline Chinese & 17 & $(9.2)$ \\
\hline India & 0 & $(0)$ \\
\hline Others & 7 & $(3.8)$ \\
\hline Marital Status & 16 & $(8.6)$ \\
\hline Single & 13 & $(7.0)$ \\
\hline Widow & 6 & $(3.2)$ \\
\hline Divorced & 150 & $(81.1)$ \\
\hline Married &
\end{tabular}

\begin{tabular}{|c|c|c|}
\hline $\begin{array}{l}\text { Table 1: Continued } \\
\text { Social Status }\end{array}$ & $(60.0)$ \\
\hline Housewife & 111 & $(29.2)$ \\
\hline Worker & 54 & $(10.8)$ \\
\hline $\begin{array}{l}\text { Self- } \\
\text { employed }\end{array}$ & 20 & \\
\hline Educational Qualification & $(3.8)$ \\
\hline Illiterate & 7 & $(7.6)$ \\
\hline Primary & 14 & $(69.2)$ \\
\hline Secondary & 128 & $(16.2)$ \\
\hline Undergraduate & 30 & $(3.2)$ \\
\hline Postgraduate & 6 & $(58.4)$ \\
\hline Economic Status & 108 & $(12.4)$ \\
\hline$<$ RM 1000 & 23 & $(21.6)$ \\
\hline $\begin{array}{l}\text { RM 1000 - } \\
\text { RM 1999 }\end{array}$ & 40 & $(7.6)$ \\
\hline RM 2000 - \\
RM 5000
\end{tabular}

\section{Knowledge Towards Osteoporosis}

Table 2 shows the responses of participants towards knowledge questions on osteoporosis. The majority $(82.7 \%)$ knew that osteoporosis causes bones to be weak and fragile (easy to break), more likely to have bone fractures $(83.8 \%)$, more likely to become shorter due to bent spine $(74.1 \%)$ and knew that they are more likely to have slouched body posture (80.5\%).

The findings also showed that they knew that they might get osteoporosis quickly if they do not drink milk and other dairy products. About $77.8 \%$ knew that they might get osteoporosis if they do not do enough exercise. Women would get osteoporosis quickly $(71.9 \%)$ and $75.1 \%$ knew that people who eat fast food and drink soft drinks regularly would get osteoporosis quickly. Regarding the ideal time to make bones strong and increase bone mass is before 25 years, $73.0 \%$ of women correctly responded to the questions.

The majority (64.3\%) knew that bone mass density is used to measure strength and quality. About $51.9 \%$ of the respondent knew that people who suffer from menopause at an early age be more likely to suffer from osteoporosis. While $49.2 \%$ knew that someone who smokes regularly would be more likely to get osteoporosis. The same score 75 (40.5\%) was achieved by the questions vegetarian and avoiding sunlight are more likely to get osteoporosis. If someone in the family already has osteoporosis, then other members are more likely to get osteoporosis, $(40.0 \%)$ of participants answered correctly. Fifty-three $(28.6 \%)$ of them do not 
know that osteoporosis affects both men and women differently. The least score was achieved by $29.7 \%$, knowing that short and thin people are more likely to get osteoporosis quickly.

Table 2: Knowledge Towards Osteoporosis $(n=185)$

\begin{tabular}{|c|c|c|c|c|c|}
\hline No. & Questions & $\begin{array}{c}\text { Yes } \\
\text { n }(\%)\end{array}$ & $\begin{array}{c}\text { No } \\
\text { n }(\%)\end{array}$ & $\begin{array}{l}\text { Don't Know } \\
\text { n (\%) }\end{array}$ & Mean (SD) \\
\hline 1 & $\begin{array}{l}\text { Osteoporosis causes bones weak and fragile (easy to } \\
\text { break). }\end{array}$ & $153(82.7)$ & $7(3.8)$ & $25(13.5)$ & $1.31(0.697)$ \\
\hline 2 & $\begin{array}{l}\text { Bone mass density (BMD) is used to measure the } \\
\text { strength and quality of bones. }\end{array}$ & $119(64.3)$ & $7(3.8)$ & $59(31.9)$ & $1.68(0.928)$ \\
\hline 3 & $\begin{array}{l}\text { The ideal time to make bones strong and increase bone } \\
\text { mass is before } 25 \text { years. }\end{array}$ & $135(73.0)$ & $18(9.7)$ & $32(17.3)$ & $1.44(0.772)$ \\
\hline 4 & Osteoporosis affects both men and women equally. & $53(28.6)$ & $108(58.4)$ & $24(13.0)$ & $1.84(0.628)$ \\
\hline \multirow[t]{11}{*}{5} & \multicolumn{5}{|l|}{ You are more likely to suffer/get osteoporosis, if } \\
\hline & $\begin{array}{l}\text { - You do not drink milk and other dairy } \\
\text { products. }\end{array}$ & $149(80.5)$ & $31(16.8)$ & $5(2.7)$ & $1.22(0.477)$ \\
\hline & - You are a vegetarian. & $75(40.5)$ & $87(47.0)$ & $23(12.4)$ & $1.72(0.673)$ \\
\hline & $\begin{array}{l}\text { - Someone in your family already has } \\
\text { osteoporosis. }\end{array}$ & $74(40.0)$ & $92(49.7)$ & $19(10.3)$ & $1.70(0.645)$ \\
\hline & - You are a female. & $133(71.9)$ & $42(22.7)$ & $10(5.4)$ & $1.34(0.577)$ \\
\hline & - $\quad$ You avoid sunlight. & $75(40.5)$ & $94(50.8)$ & $16(8.6)$ & $1.68(0.626)$ \\
\hline & $\begin{array}{l}\text { - You suffer from menopause at an early age } \\
\text { (before } 50 \text { years old). }\end{array}$ & $96(51.9)$ & $50(27.0)$ & $39(21.1)$ & $1.69(0.799)$ \\
\hline & $\begin{array}{l}\text { - You do not enough physical } \\
\text { activities/exercise everyday. }\end{array}$ & $144(77.8)$ & $36(19.5)$ & $5(2.7)$ & $1.25(0.492)$ \\
\hline & - You smoke regularly. & $91(49.2)$ & $77(41.6)$ & $17(9.2)$ & $1.60(0.653)$ \\
\hline & - You are short and thin. & $55(29.7)$ & $113(61.1)$ & $17(9.2)$ & $1.79(0.591)$ \\
\hline & $\begin{array}{ll}-\quad \text { You eat fast food and drink soft drinks } \\
\text { regularly. }\end{array}$ & $139(75.1)$ & $45(24.3)$ & $1(0.5)$ & $1.25(0.449)$ \\
\hline 6 & \multicolumn{4}{|l|}{ If you have osteoporosis, you are more likely to } & \\
\hline & - $\quad$ Have bone fractures. & $155(83.8)$ & $17(9.2)$ & $13(7.0)$ & $1.23(0.566)$ \\
\hline & - Become shorter due to bent spine. & $137(74.1)$ & $24(13.0)$ & $24(13.0)$ & $1.39(0.707)$ \\
\hline & - $\quad$ Have slouched body posture. & $149(80.5)$ & $23(12.4)$ & $13(7.0)$ & $1.26(0.581)$ \\
\hline
\end{tabular}

\section{Osteoporosis Knowledge Level}

Table 3 illustrates the frequency, percentage, mean and standard deviation of osteoporosis among women in the study. More than half of participants $(40.5 \%)$ had good knowledge level, $39.5 \%$ of them had fair knowledge level and smaller proportions had poor knowledge level (20.0\%). The participants achieved a mean \pm SD score of knowledge as $63.18( \pm 18.91)$.

Table 3: Level of Knowledge about Osteoporosis among Women $(n=185)$

\begin{tabular}{|l|c|c|}
\hline Knowledge Level & Frequency (\%) & Mean (SD) \\
\hline Good & $75(40.5)$ & \multirow{2}{*}{$63.18(18.91)$} \\
\cline { 1 - 2 } Fair & $73(39.5)$ & \multirow{1}{*}{} \\
\cline { 1 - 2 } Poor & $37(20.0)$ & \\
\hline
\end{tabular}

\section{Practice Towards Osteoporosis}

In Table 4, data is presented regarding how participants respond towards practice on osteoporosis. Almost all the participants $(89.7 \%)$ do housework by themselves. The majority of participants $(64.9 \%)$ prefer to eat vegetables. The findings also showed that $43.2 \%$ of them always take the milk and dairy products. Fifty-nine (31.9\%) of them still spend 20 minutes under sun on at least three different days while (29.7\%) always exercise for at least 30 minutes 3 times a week. Most of the $96(51.9 \%)$ participants always drink coffee, tea, or soft drinks. Fourteen (7.6\%) of them always discuss osteoporosis with family, friends, etc. whereas (4.3\%) always discuss osteoporosis with a doctor, nurse or pharmacist. None of them have a habit of smoking. Only $2(1.1 \%)$ of them still drink alcohol. 
Table 4: Practice Towards Osteoporosis ( $=185$ )

\begin{tabular}{|c|c|c|c|c|c|c|}
\hline Question & $\begin{array}{c}\text { Always } \\
(\%)\end{array}$ & $\begin{array}{l}\text { Frequently } \\
\quad(\%)\end{array}$ & $\begin{array}{c}\text { Sometimes } \\
(\%)\end{array}$ & $\begin{array}{c}\text { Rarely } \\
(\%)\end{array}$ & $\begin{array}{c}\text { Never } \\
(\%)\end{array}$ & Mean (SD) \\
\hline $\begin{array}{l}\text { I do exercise for at least } 30 \\
\text { minutes } 3 \text { times a week. }\end{array}$ & $\begin{array}{c}55 \\
(29.7)\end{array}$ & $\begin{array}{c}15 \\
(8.1)\end{array}$ & $\begin{array}{c}76 \\
(41.1)\end{array}$ & $\begin{array}{c}27 \\
(14.6)\end{array}$ & $\begin{array}{c}12 \\
(6.5)\end{array}$ & $2.60(1.234)$ \\
\hline $\begin{array}{l}\text { I take milk and d airy products } \\
\text { (egg, cheese, butter). }\end{array}$ & $\begin{array}{c}80 \\
(43.2)\end{array}$ & $\begin{array}{c}45 \\
(24.3)\end{array}$ & $\begin{array}{c}37 \\
(20.0)\end{array}$ & $\begin{array}{c}18 \\
(9.7)\end{array}$ & $\begin{array}{c}5 \\
(2.7)\end{array}$ & $2.04(1.127)$ \\
\hline $\begin{array}{l}\text { I spend } 20 \text { minutes under sun } \\
\text { on at least } 3 \text { different days. }\end{array}$ & $\begin{array}{c}59 \\
(31.9)\end{array}$ & $\begin{array}{c}31 \\
(16.8)\end{array}$ & $\begin{array}{c}36 \\
(19.5)\end{array}$ & $\begin{array}{c}28 \\
(15.1)\end{array}$ & $\begin{array}{c}31 \\
(16.8)\end{array}$ & $2.68(1.475)$ \\
\hline I prefer to eat vegetables. & $\begin{array}{c}120 \\
(64.9)\end{array}$ & $\begin{array}{c}40 \\
(21.6)\end{array}$ & $\begin{array}{c}17 \\
(9.2)\end{array}$ & $\begin{array}{c}5 \\
(2.7)\end{array}$ & $\begin{array}{c}3 \\
(1.6)\end{array}$ & $1.55(0.890)$ \\
\hline $\begin{array}{l}\text { I discuss osteoporosis with } \\
\text { family, friends, etc. }\end{array}$ & $\begin{array}{c}14 \\
(7.6)\end{array}$ & $\begin{array}{c}9 \\
(4.9)\end{array}$ & $\begin{array}{c}51 \\
(27.6)\end{array}$ & $\begin{array}{c}47 \\
(25.40\end{array}$ & $\begin{array}{c}64 \\
(34.6)\end{array}$ & $3.75(1.200)$ \\
\hline $\begin{array}{l}\text { I discuss osteoporosis with my } \\
\text { doctor, nurse or pharmacist. }\end{array}$ & $\begin{array}{c}8 \\
(4.3)\end{array}$ & $\begin{array}{c}11 \\
(5.9)\end{array}$ & $\begin{array}{c}30 \\
(16.2)\end{array}$ & $\begin{array}{c}38 \\
(20.5)\end{array}$ & $\begin{array}{c}98 \\
(53.0)\end{array}$ & $4.12(1.145)$ \\
\hline I have a habit of smoking. & 0 & 0 & 0 & $\begin{array}{c}1 \\
(0.5)\end{array}$ & $\begin{array}{c}184 \\
(99.5)\end{array}$ & $4.99(0.074)$ \\
\hline I drink alcohol. & $\begin{array}{c}2 \\
(1.1)\end{array}$ & 0 & 0 & $\begin{array}{c}3 \\
(1.6)\end{array}$ & $\begin{array}{c}180 \\
(97.3)\end{array}$ & $4.94(0.432)$ \\
\hline $\begin{array}{l}\text { I do housework by myself, e.g., } \\
\text { cleaning, cooking food, making } \\
\text { a bed etc. }\end{array}$ & $\begin{array}{c}166 \\
(89.7)\end{array}$ & $\begin{array}{c}10 \\
(5.4)\end{array}$ & $\begin{array}{c}4 \\
(2.2)\end{array}$ & 0 & $\begin{array}{c}5 \\
(2.7)\end{array}$ & $1.21(0.731)$ \\
\hline $\begin{array}{l}\text { I drink coffee, tea or soft } \\
\text { drinks. }\end{array}$ & $\begin{array}{c}96 \\
(51.9)\end{array}$ & $\begin{array}{c}26 \\
(14.1)\end{array}$ & $\begin{array}{c}27 \\
(14.1)\end{array}$ & $\begin{array}{c}29 \\
(15.7)\end{array}$ & $\begin{array}{c}7 \\
(3.8)\end{array}$ & $2.05(1.280)$ \\
\hline
\end{tabular}

\section{Osteoporosis Practice Level}

Table 5 shows only (4.3\%) of participants had a good practice, fair (42.2\%) and the majority had poor practice level $(53.5 \%)$. The mean (SD) of osteoporosis practice score was $50.18( \pm 10.79)$.

Table 5: Level of Practice About Osteoporosis among Women $(n=185)$

\begin{tabular}{|c|c|c|}
\hline Practice Level & Frequency (\%) & Mean (SD) \\
\hline & & \\
\hline Good & $80.18(10.797)$ \\
\hline Fair & $78(42.3)$ & \\
\hline Poor & $99(53.5)$ & \\
\hline
\end{tabular}

Association of Socio-demographic Characteristics with Level of Knowledge towards Osteoporosis $(\mathrm{n}=\mathbf{1 8 5})$

Table 6 showed that age $(p=0.466)$, ethnicity ( $p=0.318)$, social status $(p=0.977)$, and economic status $(p=0.705)$ were not significantly associated with osteoporosis knowledge score respectively. In contrast, ethnicity $(p=0.318)$, marital status $(p=0.063)$, and educational qualification $(p=0.70)$ were not significantly associated with osteoporosis knowledge score. 
Table 6: Association Between Socio-demographic Data and Knowledge Level Towards Osteoporosis (n=185)

\begin{tabular}{|c|c|c|c|c|c|}
\hline \multirow[t]{2}{*}{ Characteristics } & \multicolumn{3}{|c|}{ Knowledge level of Osteoporosis, n (\%) } & \multicolumn{2}{|c|}{ Pearson Chi-square } \\
\hline & Poor & Fair & Good & $X^{2}(d f)$ & $p$-value \\
\hline \multicolumn{4}{|l|}{ Age (years old) } & $3.580(4)$ & $0.466^{\mathrm{a}}$ \\
\hline $45-55$ & $22(17.7 \%)$ & $51(41.1 \%)$ & $51(41.1 \%)$ & & \\
\hline $56-65$ & $13(27.1 \%)$ & $15(31.3 \%)$ & $20(41.7 \%)$ & & \\
\hline 66 and above & $2(15.4 \%)$ & $7(53.8 \%)$ & $4(30.8 \%)$ & & \\
\hline \multicolumn{4}{|l|}{ Social status } & \multirow[t]{2}{*}{$0.459(4)$} & $0.977^{\text {a }}$ \\
\hline Housewife & $21(18.9 \%)$ & $45(40.5 \%)$ & $45(40.5 \%)$ & & \\
\hline Worker & $11(20.4 \%)$ & $21(38.9 \%)$ & $22(40.7 \%)$ & & \\
\hline Self-employed & $5(25.0 \%)$ & $7(35.0 \%)$ & $8(40.0 \%)$ & & \\
\hline \multicolumn{4}{|l|}{ Economic status } & \multirow[t]{2}{*}{$3.794(6)$} & $0.705^{\mathrm{a}}$ \\
\hline$<\mathrm{RM} 1000$ & $21(19.4 \%)$ & $46(42.6 \%)$ & $41(38.0 \%)$ & & \\
\hline RM $1000-$ RM 1999 & $6(26.1 \%)$ & $7(30.4 \%)$ & $10(43.5 \%)$ & & \\
\hline RM $2000-$ RM 5000 & $9(22.5 \%)$ & $13(32.5 \%)$ & $18(45.0 \%)$ & & \\
\hline$>$ RM 5000 & $1(7.1 \%)$ & $7(50.0 \%)$ & $6(42.9)$ & & \\
\hline \multicolumn{4}{|l|}{ Ethnicity } & \multicolumn{2}{|r|}{$0.318^{\mathrm{d}}$} \\
\hline Malay & $30(18.6 \%)$ & $67(41.6 \%)$ & $64(39.8 \%)$ & & \\
\hline Chinese & $4(23.5 \%)$ & $4(23.5 \%)$ & $9(52.9 \%)$ & & \\
\hline India & $0(0 \%)$ & $0(0 \%)$ & $0(0 \%)$ & & \\
\hline Others & $3(42.9 \%)$ & $2(28.6 \%)$ & $2(28.6 \%)$ & & \\
\hline \multicolumn{4}{|l|}{ Marital status } & \multicolumn{2}{|r|}{$0.063^{\mathrm{d}}$} \\
\hline Single & $6(37.5 \%)$ & $6(37.5 \%)$ & $4(25.0 \%)$ & & \\
\hline Widow & $5(38.5 \%)$ & $4(30.8 \%)$ & $4(30.8 \%)$ & & \\
\hline Divorced & $3(1.2 \%)$ & $1(16.7 \%)$ & $2(33.3 \%)$ & & \\
\hline Married & $23(15.3 \%)$ & $62(41.3 \%)$ & $65(43.3 \%)$ & & \\
\hline \multicolumn{4}{|l|}{ Educational qualification } & \multicolumn{2}{|c|}{$0.705^{\mathrm{d}}$} \\
\hline Illiterate & $1(14.3 \%)$ & $4(57.1 \%)$ & $2(28.6 \%)$ & & \\
\hline Primary & $3(21.4 \%)$ & $6(42.9 \%)$ & $5(35.7 \%)$ & & \\
\hline Secondary & $25(19.5 \%)$ & $51(39.8 \%)$ & $52(40.9 \%)$ & & \\
\hline Undergraduate & $8(26.7 \%)$ & $11(36.7 \%)$ & $11(36.7 \%)$ & & \\
\hline Postgraduate & $0(0 \%)$ & $1(16.7 \%)$ & $5(83.3 \%)$ & & \\
\hline
\end{tabular}

a tested by Chi-square Test

'tested by Fisher's Exact Test

*Level of significant at $p<0.05$ 
Association of Socio-demographic Characteristics with Level of Practice towards Osteoporosis

Table 7 showed that age $(p=0.348)$, ethnicity
( $p=0.855)$, marital status $(p=0.949)$, social status $(p=0.530)$, educational qualification $(\mathrm{p}=0.974)$ and economic status $(p=0.282)$ were not significantly associated with osteoporosis practice score respectively.

Table 7: Association Between Practice Level Towards Osteoporosis and Socio-demographic Data (n=185)

\begin{tabular}{|c|c|c|c|c|c|}
\hline \multirow{2}{*}{ Characteristics } & \multicolumn{3}{|c|}{ Practice level of osteoporosis } & \multicolumn{2}{|c|}{ Pearson Chi-Square } \\
\hline & Poor & Fair & Good & $X^{2}(d f)$ & $p$-value \\
\hline \multicolumn{4}{|l|}{ Age (years old) } & \multicolumn{2}{|r|}{$0.348^{\mathrm{d}}$} \\
\hline $45-55$ & $72(58.1 \%)$ & $47(37.9 \%)$ & $5(4.0 \%)$ & & \\
\hline $56-65$ & $20(41.7 \%)$ & $25(52.1 \%)$ & $3(6.3 \%)$ & & \\
\hline 66 and above & $7(53.8 \%)$ & $6(46.2 \%)$ & $0(0 \%)$ & & \\
\hline \multicolumn{4}{|l|}{ Ethnicity } & \multicolumn{2}{|r|}{$0.855^{\mathrm{d}}$} \\
\hline Malay & $85(52.8 \%)$ & $68(42.2 \%)$ & $8(5.0 \%)$ & & \\
\hline Chinese & $9(52.9 \%)$ & $8(47.1 \%)$ & $0(0 \%)$ & & \\
\hline India & $0(0 \%)$ & $0(0 \%)$ & $0(0 \%)$ & & \\
\hline Others & $5(71.4 \%)$ & $2(28.6 \%)$ & $0(0 \%)$ & & \\
\hline \multicolumn{3}{|l|}{ Marital status } & \multicolumn{2}{|l|}{0.949} & \multirow[t]{2}{*}{$0.949^{d}$} \\
\hline Single & $10(62.5 \%)$ & $6(37.5 \%)$ & $0(0 \%)$ & & \\
\hline Widow & $6(46.2 \%)$ & $7(53.8 \%)$ & $0(0 \%)$ & & \\
\hline Divorced & $3(50.0 \%)$ & $3(50.0 \%)$ & $0(0 \%)$ & & \\
\hline Married & $80(53.5 \%)$ & $78(42.2 \%)$ & $8(5.3 \%)$ & & \\
\hline \multicolumn{4}{|l|}{ Social status } & \multirow{2}{*}{\multicolumn{2}{|c|}{$0.530^{\mathrm{d}}$}} \\
\hline Housewife & $62(55.9 \%)$ & $44(39.6 \%)$ & $5(4.5 \%)$ & & \\
\hline Worker & $29(53.7 \%)$ & $22(40.7 \%)$ & $3(5.6 \%)$ & & \\
\hline Self-employed & $8(40.0 \%)$ & $12(60.0 \%)$ & $0(0 \%)$ & & \\
\hline \multicolumn{4}{|l|}{ Educational qualification } & \multicolumn{2}{|r|}{$0.974^{\mathrm{d}}$} \\
\hline Illiterate & $4(57.1 \%)$ & $3(42.9 \%)$ & $0(0 \%)$ & & \\
\hline Primary & $8(56.1 \%)$ & $6(42.9 \%)$ & $0(0 \%)$ & & \\
\hline Secondary & $68(53.1 \%)$ & $53(41.4 \%)$ & $7(5.5 \%)$ & & \\
\hline Undergraduate & $17(56.7 \%)$ & $12(40.0 \%)$ & $1(3.3 \%)$ & & \\
\hline Postgraduate & $2(33.3 \%)$ & $4(66.7 \%)$ & $0(0 \%)$ & & \\
\hline \multicolumn{4}{|l|}{$\begin{array}{l}\text { Table 7: Continued } \\
\text { Economic status }\end{array}$} & \multicolumn{2}{|r|}{$0.282^{\mathrm{d}}$} \\
\hline$<\mathrm{RM} 1000$ & $58(53.7 \%)$ & $45(41.7 \%)$ & $5(4.6 \%)$ & & \\
\hline $\begin{array}{l}\text { RM } 1000- \\
\text { RM } 1999\end{array}$ & $15(65.2 \%)$ & $8(34.8 \%)$ & $0(0 \%)$ & & \\
\hline $\begin{array}{l}\text { RM } 2000- \\
\text { RM } 5000\end{array}$ & $22(55.0 \%)$ & $17(42.5 \%)$ & $1(2.5 \%)$ & & \\
\hline$>$ RM 5000 & $4(28.6 \%)$ & $8(57.1 \%)$ & $2(14.3 \%)$ & & \\
\hline
\end{tabular}

${ }^{d}$ Fisher's Exact Test

*Level of significant at $p<0.05$ 


\section{DISCUSSION}

The majority of the participants within the age range of $45-55$ years $(67.0 \%)$, most of them were Malay $(87.0 \%)$, married $(81.1 \%)$ and housewives $(60.0 \%)$. They had an education level up to secondary education $(69.2 \%)$. The study had shown most (58.4\%) of the economic status were less than RM 1000. Based on the study results, socio-demographic characteristics (age, ethnicity, marital status, social status, educational status, economic status) had no significant association with knowledge and practice.

Such findings are comparable to the analysis by Khan et al., (2014), who documented no substantial difference between the level of education, race, marital status, and level of information. In the relationship between economic status and information level, which is in line with the present research, the Puttapitakpong et al. (2014) study also showed no statistical significance. There was also no correlation between age, race, marital status, social status, educational status, economic status, and level of practise for osteoporosis in the current study findings. The results are similar to a previous study (Khan et al., 2014), which recorded no substantial difference in osteoporosis practise among women between educational level and marital status.

The results also showed no substantial difference between gender, ethnicity, and household income, similar to the Leng, Ali \& Yusof (2017) report. The possible reason for the absence of a connection between socio-demographic data and expertise and practise is due to the geographical distribution and the setting of the analysis. The bulk of the participants studied in a tertiary teaching hospital were Malaysians. Most of the women were married, aged 45 years and over. As many of them were not employed or were housewives, the majority of women had a low level of economic status.

In this study, osteoporosis was identified among 185 respondents $(40.5 \%)$. Most participants had a strong awareness level (40.5\%), with an average score of $63.18 \%$ out of a total score of 100 . About $53.5 \%$ of participants, on the other hand, had low levels of practise. This finding was similar to the result shown by Puttapitakpong et al., (2014) and Khan et al., (2014), who reported high osteoporosis levels among the studied participants. This outcome may be because the participants in such studies have been exposed to knowledge of osteoporosis for long.
This research discovered a high level of knowledge of osteoporosis, contradicting studies performed by De Silva et al., (2014), Barzanji et al., (2013) and Fenech et al., (2011), which documented a low level of knowledge of osteoporosis among the population studied. As the populations involved in previous research were new medical entrants and unemployed with minimal experience in osteoporosis, the findings can be clarified. Some participants in the current study are unable to understand that family inheritance, menopause, smoking, and a small body frame can also cause bone density loss and contribute to osteoporosis. The results of the study are in accordance with another study done by Leng et al., (2017).

In this report, nearly half of the participants (53.5\%) had bad osteoporosis practise among 185 participants, which is comparable to the Jalili, Nakhaee, Askari \& Sharifi (2007) survey, where practise score results showed the most problematic situation compared to the two other domains (knowledge and attitude). The current research also results close to the findings of Alshammari (2014), who reported low scores for osteoporosis-related activities and found that only $42.8 \%$ of women in Saudi Arabia exercised successfully. The De Silva et al., (2014) study also recorded low levels of osteoporosis preventive practises, with just $13.6 \%$ of women participating in the prescribed exercises in Sri Lanka.

Due to their lifestyle, the results of the present study align with those of the normal practise of weightbearing activities. Participants in the present study were adults aged 45 years and older, and most were housewives interested in taking care of the lives of children. Lack of time has been one of the factors for limited time given to exercise. Lower weight-bearing practises may also be impaired by impairments in body mobility and diseases, especially in older age groups of 50 years or older.

\section{CONCLUSION}

As is seen from the present study higher percentage of women with insufficient awareness (40.5\%) and almost half $(53.5 \%)$ of research participants have poorly practised preventive assessment of osteoporosis. This research has demonstrated that the perception and practise of osteoporosis by women requires attention. Strategies are needed to increase the level of awareness 
and practise of women regarding the prevention of osteoporosis. The vulnerability and severity of the impact of osteoporosis can be experienced by women who are known in this regard.

\section{Conflicts of Interest}

The authors declare no conflicts of interest.

\section{ACKNOWLEDGMENT}

The authors would like to thank the Human Research Ethics Committee of Universiti Sains Malaysia, and Director of Hospital USM for permitting to conduct the study and for all the nurses who have been very cooperative during data collection.

\section{REFERENCES}

Abd-Alhameed, I., Saba, E. \& Darwish, H.M. (2010). Prevalence and awareness of osteoporosis among postmenopausal Palestinian women. Archives of Osteoporosis, 5(1-2), pp 111-118.

Ahmad, M.S., Mohamed, I. N., Mokhtar, S.A. \& Shuid, A.N. (2014). Review of the risk factor of osteoporosis in the Malaysian population. $R U M e s, 3, \mathrm{pp} 77-82$.

Alshammari, K.F. (2014). Women knowledge, attitude and practices about osteoporosis prevention "Riyadh Saudi Arabia". World Journal of Medical Sciences, 11(3), pp 422-431.

Barzanji, A.T., Alamri, F. A. \& Mohamed, A.G. (2013). Osteoporosis: a study of knowledge, attitude and practice among adults in Riyadh, Saudi Arabia. Journal of Community Health, 38(6), pp 1098-1105.

Bilal, M., Haseeb, A., Merchant, A. Z., Rehman, A., Arshad, M. H., Malik, M., Rehman, A. H. U., Rani, P., Farhan, E., Rehman, T.S., Shamsi, U. S. \& Sadia, A. (2017). Knowledge, beliefs and practices regarding osteoporosis among female medical school entrants in Pakistan. Asia Pacific Family Medicine, 16(1), pp.1-7.

De Silva, R. E. E., Haniffa, M. R., Gunathillaka, K. D. K., Atukorala, I., Fernando, E. D. P. S. \& Perera, W. L. S. P. (2014). A descriptive study of knowledge, beliefs and practices regarding osteoporosis among female medical school entrants in Sri Lanka. Asia Pacific Family Medicine, 13(1), pp 15.

Fenech, J., Galea, R., Serracino-Inglott, A. \& Azzopardi, L.M. (2011). Management and patient knowledge of osteoporosis. Journal of Applied Therapeutic Research, 8, pp 101-106.

International Osteoporosis Foundation (2017). Impact of Osteoporosis. Retrieved from: https://www.iofbonehealth. org/impact-osteoporosis

Jalili, Z., Nakhaee, N., Askari, R. \& Sharifi, V. (2007). Knowledge, Attitude and Preventive Practice of Women Concerning Osteoporosis. Iranian Journal of Public Health, 36(2), pp 19-24.

Khan, Y. H., Sarriff, A., Khan, A. H. \& Mallhi, T. H. (2014). Knowledge, attitude and practice (KAP) survey of osteoporosis among students of a tertiary institution in Malaysia. Tropical Journal of Pharmaceutical Research, 13(1), pp 155-162.

Leng, L. S., Ali, A. \& Yusof, H. M. (2017). Knowledge, Attitude and Practices towards Osteoporosis Prevention among Adults in Kuala Lumpur, Malaysia. Malaysian Journal of Nutrition, 23(2), pp 279-290.

Martin, A. R., Sornay-Rendu, E., Chandler, J. M., Duboeuf, F., Girman, C. J. \& Delmas, P. D. (2002). The impact of osteoporosis on quality-of-life: the OFELY cohort. Bone, 31(1), pp 32-36.

National Osteoporosis Foundation (2015). Fast facts on osteoporosis. Retrieved from: https://cdn.nof.org/wpcontent/uploads/2015/12/Osteoporosis-Fast-Facts.pdf

Puttapitakpong, P., Chaikittisilpa, S., Panyakhamlerd, K., Nimnuan, C., Jaisamrarn, U. \& Taechakraichana, N. (2014). Inter-correlation of knowledge, attitude, and osteoporosis preventive behaviors in women around the age of peak bone mass. BMC Women's Health, 14(1), pp 35. 
Samia, A., Hejar, A.R., Suriani, I. \& Emilia, Z.A. (2017). An overview of osteoporosis and heath promotional strategies for community-based osteoporosis prevention in Malaysia. International Journal of Public Health and Clinical Sciences, 4(1), pp 28-40.

Saw, S. M., Hong, C. Y., Lee, J., Wong, M. L., Chan, M. F., Cheng, A. \& Leong, K. H. (2003). Awareness and health beliefs of women towards osteoporosis. Osteoporosis International, 14(7), pp 595-601.

Terrio, K. \& Auld, G. W. (2002). Osteoporosis knowledge, calcium intake, and weight-bearing physical activity in three age groups of women. Journal of Community Health, 27(5), pp 307-320.

Tümay, S., Lale, Ö. \& Nursel, C.B. (2017). An overview and management of osteoporosis. European Journal of Rheumatology, 4(1), pp 46-56.

Vujasinovic-Stupar, N., Radojcic, L., Tadic, I. \& Pavlov-Dolijanovic, S. (2017). Osteoporosis-related knowledge among Serbian postmenopausal women. Vojnosanitetski pregled, 74(5), pp 445-449.

World Health Rankings (WHO) (2018). Life expectancy in Malaysia. Retrieved from: https://www. worldlifeexpectancy.com/malaysia-life-expectancy. 